\title{
Transitional Feedback Schedules during Computer-Based Problem-Solving Practice
}

\author{
Amy M. Johnson, Jana Reisslein, and Martin Reisslein \\ School of Electrical, Computer, and Energy Engineering, \\ Arizona State University, Tempe, AZ 85287, USA
}

Correspondence should be addressed to Martin Reisslein, Arizona State University, School of Electrical, Computer, and Energy Engineering, P.O. Box 875706, Tempe, AZ 85287-5706, USA. Email: reisslein@asu.edu; Telephone: 480-965-8593; Fax: 480-965-8325 


\title{
Transitional Feedback Schedules during Computer-Based Problem-Solving Practice
}

\begin{abstract}
Feedback has a strong influence on effective learning from computer-based instruction. Prior research on feedback in computer-based instruction has mainly focused on static feedback schedules that employ the same feedback schedule through an instructional session. This study examined transitional feedback schedules in computer-based multimedia instruction on procedural problem-solving in electrical circuit analysis. Specifically, we compared two transitional feedback schedules: the TFS-P schedule switched from initial feedback after each problem step to feedback after a complete problem at later learning states; the TFP-S schedule transitioned from feedback after a complete problem to feedback after each problem step. As control conditions, we also considered two static feedback schedules, namely providing feedback after each practice problem-solving step (SFS) or providing feedback after attempting a complete multi-step practice problem (SFP). Results indicate that the static stepwise (SFS) and transitional stepwise to problem (TFS-P) feedback produce higher problem solving near-transfer post-test performance than static problem (SFP) and transitional problem to step (TFP-S) feedback. Also, TFS-P resulted in higher ratings of program liking and feedback helpfulness than TFP-S. Overall, the study results indicate benefits of maintaining high feedback frequency (SFS) and reducing feedback frequency (TFS-P) compared to low feedback frequency (SFP) or increasing feedback frequency (TFPS) as novice learners acquire engineering problem solving skills.
\end{abstract}

Keywords: Delayed feedback; Immediate feedback; Feedback sequencing; Practice problem; Problem Solving 


\section{Introduction}

Feedback is widely recognized as one of the most powerful influences on student learning (Kulhavy \& Stock, 1989; Mory, 2004; Shute, 2008). Broadly defined as “information provided by an agent (e.g., teacher, peer, book, parent, self, or experience) regarding aspects of one's performance or understanding” (Hattie \& Timperley, 2007, p. 81), feedback is used to update students on how well a task has been completed and how performance can be improved. Results from a set of 12 meta-analyses indicate that feedback is among the top five influences on student achievement (Hattie, 1999). Although feedback has been widely examined in classroom settings (Bangert-Drowns, Kulik, Kulik, \& Morgan, 1991), typically with a teacher as the agent of feedback, meta-analyses conducted by Kluger and Denisi (1996) indicated computerized feedback actually produced stronger effect sizes than other modes of feedback (Cohen's $\bar{d}_{\text {comp }}=0.41 ; \bar{d}_{\text {other }}=0.23$ ). In contrast to teacher-led instruction to an entire class, computer-based instruction where each student has his/her own computer provides each student individualized feedback and allows each student to individually pace the progression through the lesson (Schoppek \& Tulis, 2010; Yang, et al., 2012).

Computer-based learning environments have the capability to generate a variety of feedback mechanisms to inform learners about task performance (Corbalan, Paas, \& Cuypers, 2010; Hsieh, \& O’Neil, 2002; Narciss, et al., 2013). The most basic form of feedback is knowledge of results (KOR), or corrective feedback, which simply reports whether a studentgenerated response is correct or incorrect (Clark \& Dwyer, 1998; Mason \& Bruning, 2001). Although this form of feedback can assist learners in evaluating their success rate, it does not provide principle-based explanations for why responses are correct or incorrect (Moreno, 2004; Moreno \& Mayer, 2007). Consequently, corrective feedback is often coupled with explanatory feedback which, in problem-solving, often provides the correct answer, along 
with the procedure used to obtain that answer. Moreno and Mayer (2007) offered the feedback design principle: "Students learn better with explanatory rather than corrective feedback alone." According to Moreno and Mayer (2007) the utilization of explanatory feedback reduces unnecessary demands on limited cognitive resources by offering meaningful explanations to repair students' misconceptions. Consequently, the present study employed feedback in the form of combined corrective and explanatory feedback throughout.

In addition to the form of feedback, the timing (schedule) of the feedback can potentially influence its effectiveness (Kulik \& Kulik, 1988; Mathan \& Koedinger, 2003). The present study examines transitional feedback schedules that vary the feedback schedule as the learner progresses through computer-based instruction. The following Section 1.1 briefly describes the cognitive load theory. The subsequent Section 1.2 reviews related research on static feedback schedules that maintain the same feedback timing throughout instruction, while existing research and concepts surrounding transitional feedback schedules are presented in Section 1.3. Based on the background presented in Sections 1.1-1.3, hypotheses for transitional feedback schedules are presented in Section 1.4.

\subsection{Brief Overview of Cognitive Load Theory}

Cognitive load theory (CLT; Sweller, van Merrienboer, \& Paas, 1998) is based on the widely accepted assumption that working memory capacity is limited (Baddeley, 1986). According to CLT, every instructional condition imposes a load on working memory which is subdivided into three types: 1) intrinsic cognitive load; 2) extraneous cognitive load; and 3) germane cognitive load. The amount of intrinsic load imposed is dependent on the degree of element interactivity (i.e., the number of elements which need to be processed simultaneously) of the information to be learned. Germane load relates to the active cognitive processes which contribute to the construction of mental representations (i.e., schemas). Extraneous load does not contribute to learning, and if working memory capacity is entirely 
occupied by intrinsic and extraneous processing, no cognitive resources will be available to enact germane processes. In such cases, although a learner may successfully complete a learning task, he or she will not be able to construct and automate schemas and little learning will occur.

Once a learner has developed domain-relevant schemas, individual elements are chunked in long-term memory (Anderson, 1993; Laird, Rosenbloom, \& Newell, 1985) and intrinsic cognitive load is decreased for learning tasks within the same domain (Renkl \& Atkinson, 2003). In problem-solving specifically, as expertise develops, learners obtain procedural knowledge which permits quick problem solving with little mental effort (Anderson, Fincham, \& Douglas, 1997). Consequently, in later stages of learning, more cognitive resources are available for germane processes related to schema automation, and learners can process larger amounts of information simultaneously.

A significant challenge of computer-based instruction is to promote meaningful learning by increasing students' active processing of the instructional materials while reducing cognitive load (Sweller, 1999; Sweller, van Merrienboer, \& Paas, 1998). Therefore, according to CLT, it is necessary to carefully examine the relationships between the cognitive demands imposed by the learning environment, the learner's expertise level, and the desired learning outcomes. Worked examples that provide all problem solving steps worked out (solved) for the learner are a widely studied instructional design strategy for reducing cognitive demands in instruction on problem solving (Atkinson, Derry, Renkl, \& Wortham, 2000; Biesinger \& Crippen, 2010). The effects of different sequences (schedules) of worked examples and practice problems, that are to be solved by the learner, have been examined in several studies (e.g., Leppink, Paas, van Gog, van der Vleuten, \& van Merriënboer, 2014; Van Gog, Kester, \& Paas, 2011). Transitioning from worked examples to practice problems has generally been found to better foster learning for novices than transitioning from practice 
problems to worked examples. Akin to comparing the transitioning from either worked examples to practice problems with transitioning from practice problems to worked examples (Leppink, et al., 2014; Van Gog, et al., 2011), we compare the transitioning from either feedback for each practice problem step to feedback for an entire practice problem with transitioning from feedback for an entire practice problem to feedback after each problem step in the present study.

\subsection{Static Feedback Schedules}

\subsubsection{Immediate Feedback}

According to cognitive load theory, immediate feedback in problem-solving instruction presents advantages to the novice learner (Sweller, van Merrienboer, \& Paas, 1998). It increases the likelihood of a student making meaningful connections between his/her answer for a given problem step and the corresponding feedback information because both pieces of information can be simultaneously processed in working memory. Immediate feedback also helps avoid cognitive overload by focusing on problem-solving sub-goals, i.e., individual problem solving steps.

Past research suggests that novice learners benefit from just-in-time information to repair or correct errors immediately after attempting an individual problem-solving step, as for instance Kester, Kirschner, and van Merriënboer (2006) found in their study on troubleshooting specific issues in electrical circuits. In their review of studies comparing immediate vs. delayed feedback, Kulik and Kulik (1988) found that, although delayed feedback is more beneficial for direct recall of test content, immediate feedback is more effective for developing knowledge needed to apply learning to novel questions. Reviews by Azevedo and Bernard (1995) as well as Mason and Bruning (2001) have similarly found benefits of immediate feedback for learning problem solving. Studies by Dihoff, Brosvic, Epstein, and Cook (2004) for the domain of preparation for general factual knowledge tests, 
and recently by Moreno, Reisslein, and Ozogul (2009) in the engineering problem solving domain, as well as Lin, Lai, and Chuang, (2013) on database concepts found also that immediate (timely) feedback after individual problem steps leads to improved learning compared to delayed feedback that is given after a learner has attempted to solve an entire multi-step problem. Van der Kleij, Eggen, Timmers, and Veldkamp (2012) in their examination of students learning economics facts found that students paid closer attention to immediate feedback than to delayed feedback.

\subsubsection{Delayed Feedback}

Several studies have found benefits of delayed feedback over immediate feedback for specific learning tasks. For instance, for learning computer programming, Schooler and Anderson (2008) found that immediate feedback led to more errors on posttest problems than delayed feedback after the students had attempted more steps. Similarly, delayed feedback led to better detection of errors than immediate feedback in an adventure game (Lewis \& Anderson, 1985). Munro, Fehling, and Towne (1985) found lower error rates for learning an air intercept controller task with delayed feedback compared to immediate feedback. Delayed feedback can also lead to better decision making in organizational management tasks (Lam, DeRue, Karam, \& Hollenbeck, 2011; Lurie \& Swaminathan, 2009). These existing studies indicate benefits of delayed feedback for the learning of tasks that involve strategic planning and processing. More generally, the learning of effective task strategies has been found to be best supported by process-oriented feedback that focuses on the underlying solution processes, rather than specific outcomes of solution attempts (Butler 1987; Earley, Northcraft, Lee, \& Lituchy, 1990; Johnson, Perlow, \& Pieper, 1993).

Delayed feedback has also shown advantages for learning factual knowledge (Butler \& Roediger, 2008; Smith \& Kimball; 2010) as well as motor skill development (Swinnen, Schmidt, Nicholson, \& Shapiro; 1990), which is considered in more detail in Section 1.3. 
Experiments for category learning (Maddox, Ashby, \& Bohil, 2003; Maddox \& Ing, 2004) indicated a complex pattern of effects of immediate vs. delayed feedback depending on the specific type of category learning. Timing of feedback in learning various domains has been extensively examined, but typically these studies considered static feedback schedules throughout a learning session.

\subsection{Transitional Feedback Schedules}

Research on transitional feedback schedules has so far focused on the training domains of motor skills, organizational management, and military missions. In the domain of motor skill development, multiple studies (e.g., Butki \& Hoffman, 2003; Ho \& Shea, 1978; Winstein \& Schmidt, 1990; Wulf \& Schmidt, 1989) discovered that a transitional feedback schedule that initially gives feedback after each practice trial and later gives feedback only after several practice trials improves development of the motor skill compared to a static feedback schedule. However, this finding does not readily extend to complex motor skills (Wulf \& Shea, 2002).

Reducing (fading) the level of detail of feedback has been compared with increasing the level of detail of feedback during training for management decisions in a simulated factory (Goodman \& Wood, 2009; Goodman, Wood, \& Chen, 2011). The pattern of results indicated that fading feedback led to positive management decisions, whereas increasing feedback led to a broader exposure of positive and negative management decisions.

Four combinations of sequences of detailed process-oriented feedback and summative outcome-oriented feedback have been compared in military training on aircraft monitoring (Van Duyne, et al., 2001). No significant differences among the different sequencing conditions were found. Billings (2012) compared static detailed feedback and static general feedback with feedback that adaptively transitioned from detailed to general when trainees reached a prescribed competence level, as well as with feedback that was initially general and 
remained general or transitioned to detailed feedback depending on the trainees performance in training for simulated military missions. The adaptive detailed-to-general feedback transition led to faster increase in competence; however at the end of the training, static detailed feedback performed equivalently to the two transitioning conditions.

The present study extends the research on transitional feedback schedules to computer-based learning of procedural problem solving. Novices learned parallel electrical circuit analysis through practicing multi-step problem solving in a multimedia module.

\subsection{Goals of the Experiment and Study Hypotheses}

The reported experiment investigated the impact of different feedback schedules using four experimental conditions: Static feedback stepwise (SFS), which provided immediate feedback on each problem step across all practice; Static feedback problem (SFP), which provided summative feedback on entire multi-step problems across all practice; Transitional feedback stepwise - problem (TFS-P), which provided stepwise feedback in the first half of practice and summative feedback in the second half of practice; and transitional feedback problem - stepwise (TFP-S), which provided summative feedback in the first half of practice and stepwise feedback in the second half of practice. Following the feedback design principle (Moreno \& Mayer, 2007), all experimental conditions utilized corrective and explanatory feedback. The effect of the different feedback schedules was examined using practice problem performance and a near-transfer problem-solving post-test, as well as learner self-reports on the liking of the program, feedback helpfulness, and perceived cognitive load.

Based on the conflicting research results on immediate and delayed feedback, as reviewed in Sections 1.2.1 and 1.2.2 as well as the reviewed research on transitional feedback schedules in Section 1.3, we formulate the following hypotheses for the present study.

\subsubsection{Feedback Fading Hypothesis}


The feedback fading hypothesis posits that the TFS-P feedback gives the highest learning performance of multi-step engineering problem solving, most positive learning perceptions, and lowest cognitive load among the four compared feedback schedules. The TFS-P feedback initially supports the learner by providing immediate feedback on the specific outcome of each individual problem step. In early stages of learning, students may require extra instructional support (i.e., scaffolding in the form of stepwise feedback) to construct fundamental knowledge structures related to solving individual problem steps. Furthermore, beginning learners may not be able to process cumulative (summative) feedback because of high element interactivity (Sweller, van Merrienboer, \& Paas, 1998). As the students' problem-solving experience increases and relevant schemas are acquired, stepwise feedback may be superfluous, and the instructional support can be faded. At this point, learning may be optimized when feedback is provided less often and encompasses a larger number of problem-solving elements, i.e., summative feedback on an entire problem, so as to support the learning of the overall solution strategy for the multi-step problems.

\subsubsection{Inverse Feedback Fading Hypothesis}

The inverse feedback fading hypothesis posits that the TFP-S feedback gives the highest learning performance of multi-step engineering problem solving, most positive learning perceptions, and lowest cognitive load among the four compared feedback schedules. The TFP-S feedback initially supports the learner by providing summative feedback on the entire problem so as to support the development of solution strategies and processes for the multi-step problems. Prior empirical studies reviewed in Section 1.2.2 have found that delayed (summative) feedback has benefits for tasks involving high degrees of strategic planning and processing. The delayed (summative) feedback in the initial learning stages may foster the acquisition of the overall solution strategy. Once the learner has acquired the overall solution strategy, specific step-wise feedback immediately after each 
problem step may aid in refining the individual solution steps within the overall solutions strategy.

\section{Method}

\subsection{Participants}

Participants included 156 students enrolled in a local public Southwestern U.S. high school, 71 females (45.5\%) and 85 males $(54.5 \%)$. The participants were novices to the domain of electrical circuit analysis. The mean age of the participants was 14.6 years $(S D=$ 0.96 years). Seventy-one (45.5\%) of participants reported they were Caucasian, 58 (37.2\%) reported they were Hispanic American, 13 (8.3\%) reported they were African American, 7 (4.5\%) reported they were Native American, 5 (3.2\%) reported they were Asian American, and $2(1.3 \%)$ reported being of Other ethnicity. Participants were randomly assigned to experimental conditions. There were 38 participants in the static feedback stepwise (SFS) condition, 38 in the static feedback problem (SFP) condition, 41 in the transitional feedback stepwise - problem (TFS-P) condition, and 39 in the transitional feedback problem stepwise (TFP-S) condition.

\subsection{Computerized Materials}

All participants completed an interactive computerized program consisting of the following elements: 1) a demographic questionnaire in which participants reported their gender, age, and ethnicity; 2) a computerized pre-test; 3) an instructional session that presented an introduction to electric circuit analysis; 4) a problem-solving practice session, including four electric circuit analysis problems; and 5) a program rating questionnaire. The program logged time on task for each element.

\subsubsection{Pre-test}

A computerized pre-test consisting of six multiple-choice questions was used to assess students' domain-relevant prior knowledge (with internal reliability of $\alpha=.63$ ). The pre-test questions covered elementary algebra, which is an important relevant prior 
knowledge area for elementary electrical circuit analysis (McDermott \& Shaffer, 1992; Irwin \& Nelms, 2008; Reisslein, Moreno, \& Ozogul, 2010), as well as prior knowledge of resistive electrical circuits. Each correctly answered question was assigned one point, resulting in a maximum pre-test score of 6 points. The average performance on the pre-test indicated low prior domain knowledge $(M=2.67 ; S D=1.26)$.

\subsubsection{Instructional Session}

The instructional program included a brief introduction to electrical circuit analysis. This section provided students the definitions and units of electrical current, voltage, and resistance. During this segment of instruction, learners were also shown the procedure for calculating the total resistance of a parallel circuit when given source voltage and individual resistance values, using Ohm's Law. Three steps were demonstrated: (i) note that the voltage $(V)$ is the same over each individual resistor and calculate the value of the current $(I)$ flowing through each individual resistor using Ohm's Law (e.g., $I_{1}=V / R_{1}$ ), (ii) calculate the total current $\left(I_{\text {Total }}\right)$ flowing in the circuit by summing up the currents flowing through the individual resistors $\left(I_{\text {Total }}=I_{1}+I_{2} \ldots\right)$, and (iii) calculate the total resistance $\left(R_{\text {Total }}\right)$ of the parallel circuit by applying Ohm's Law $\left(R_{\text {Total }}=V / I_{\text {Total }}\right)$. The introduction was identical for all experimental conditions. Throughout, the instructional and practice sessions, the variables representing electrical voltage, current, and resistance were color coded, as illustrated in the sample screen shot in Fig. 1, to aid with selecting, organizing, and integrating the circuit representations (Reisslein, Johnson, \& Reisslein, 2014; Skromme, et al., 2013).

\subsubsection{Practice session}

The practice session presented learners with four isomorphic electrical circuit problems in which students were asked to compute the total resistance of a parallel electrical circuit. For each practice problem, the learner had to use the knowledge demonstrated in the introduction in order to solve the problem, using the three problem-solving steps described 
above. The practice session was self-paced. Learners completed each solution step and received corrective and explanatory feedback to the solution steps according to the experimental condition.

In the static feedback stepwise (SFS) condition, for each practice problem, the problem solution steps (e.g., calculate the individual current flows in step 1) were presented consecutively. That is, for each problem solving step, a prompt was presented asking the learner for a solution attempt of the step, see illustration in Fig. 1 for the prompt of the first solution step. The learner received corrective and explanatory feedback to each solution step after he/she had attempted the step and pressed the 'Continue' button. If the solution attempt was correct, the feedback confirmed the correctness of the solution. If the solution attempt was incorrect, the feedback noted that the attempt was incorrect and presented how to solve the step correctly as well as the correct solution, as illustrated in Fig 2 for the first step of a practice problem. In the screen layout illustrated in Fig. 2, the feedback for a given step appeared within the "box" for the step, whereby the correct solution step was positioned just to the right of the entry fields for the solution attempt. In addition, the equations representing the correct solution of the step were integrated into the circuit diagram as such integration may reduce the split attention effect (Ginns, 2006; Ozogul, Johnson, Moreno, \& Reisslein, 2012). After studying the feedback, the learner could click on the "Continue" button to proceed to the next solution step while the correct solution for the preceding step remained on the screen. Figure2 illustrates the learning environment at the instant after the learner had clicked "Continue" to proceed to the second solution step of a sample practice problem.

In the static feedback problem (SFP) condition, for each practice problem, the learner was consecutively presented with the prompts for the individual solution steps. That is, when the learner had attempted a step and pressed "Continue", s/he was immediately presented with the prompt for the next solution step, as illustrated in Fig. 3. When the learner pressed 
the "Continue" button after having attempted the third problem step, s/he received corrective and explanatory feedback for all problem steps, whereby the feedback had exactly the same form and provided exactly the same information as in the SFS condition.

In the transitional feedback stepwise - problem (TFS-P) condition, learners attempted and received feedback on the first two practice problems as in the SFS condition and attempted and received feedback on the last two practice problems as in the SFP condition. In the transitional feedback problem - stepwise (TFP-S) condition, the first two practice problems followed the SFP condition and the last two practice problems followed the SFS condition. See Table 1 for a representation of the feedback schedule for each condition.

The computer module logged the practice session performance for each participant. For scoring the practice session performance, one point was assigned for each correctly solved practice problem step. In order to obtain insights into the impact of the feedback transition, we consider the number of correctly solved steps in the first two practice problems (P1 and P2 with an aggregate maximum score of 6), and the number of correctly solved steps in the last two practice problems (P3 and P4 with total maximum score of 6). Considering the aggregate score for P1 and P2 as well as the aggregate score for P3 and P4 allowed for examination of the different feedback schedules, i.e., stepwise feedback or problem (summative) feedback, within a transitional feedback condition.

\subsubsection{Program rating questionnaire}

The last section of the computer program was a program rating questionnaire. Six Likert-type items asked participants to rate their learning perceptions on a 5-point scale, from 1--strongly disagree to 5--strongly agree. Two items related to overall liking of the computer program ("I liked the lesson"; "I enjoyed learning with the lesson"); two items related to the helpfulness of the feedback ("The feedback in the lesson helped me learn"; "The feedback helped me to solve the problems"); two items assessed perceived cognitive load ("The lesson 
was difficult"; "Learning the material in the lesson required a lot of effort"). The internal reliability of the questionnaire was examined using Cronbach's alpha. The two 'Program Liking' items had an internal validity of .89; the two 'Feedback Helpfulness' items had an internal validity of .92; the two 'Cognitive Load' items had an internal validity of .79.

\subsection{Paper and pencil materials}

The paper-based near-transfer post-test was designed to assess students' ability to transfer the problem-solving skills they obtained during instruction to an isomorphic set of problems. The post-test was printed in black font on white paper. Students solved four novel electric circuit problems with the same underlying structure as the problems used in the practice session, but with different surface characteristics. A sample post-test problem was: "You have a parallel circuit with three resistors; $R_{1}=4.5 \mathrm{Ohm}, R_{2}=18 \mathrm{Ohm}$, and $R_{3}=72$ Ohm. The resistors are connected to a voltage source with $V=9$ Volt. What is the total resistance of this parallel electrical circuit?" Each problem included the three problemsolving steps described above, and was scored by assigning one point for each correct solution step (i.e., $\max$ score for each problem $=3$; $\max$ score for post-test $=12$ ). The posttest was scored independently by two engineering experts (inter-rater reliability $=98.4 \%$ ). The internal reliability of the post-test items was $\alpha=.77$.

\subsection{Apparatus}

The computer-based instructional module was developed with Adobe Flash CS3 software, an authoring tool for web-based and standalone multimedia programs. Adobe Flash provides an interactive visual programming platform that can flexibly import different types of images, videos, and sounds. The diversity of Adobe Flash facilitated the development of a visually pleasing module that was easy to alter for the different experimental conditions. Each segment of the instruction was narrated and a diagram of the parallel electrical circuit under consideration was displayed in the top half of the screen. The diagram was represented with 
conventional abstract electrical engineering symbols (Johnson, Reisslein, \& Reisslein, 2013; Mason, et al., 2013; Reisslein, et al., 2010). The prompts for entering the solution attempts and the corresponding corrective and explanatory feedback (Moreno, 2004; Moreno \& Mayer, 2007; Moreno, et al., 2009) were displayed in the bottom half of the screen.

Adobe Flash permits for traditional programming using Flash's language "ActionScript". Through ActionScript we controlled the different feedback schedules. ActionScript command codes checked the solution attempts of the learners and provided appropriate feedback. ActionScript command codes also logged learner interaction data and controlled the elements displayed in each screen.

The apparatus consisted of a set of laptop computer system, with a screen resolution of $1,680 \times 1,050$ pixels, and headphones.

\subsection{Procedure}

Students participated in the experiment during a regular class session in their normal classroom. Each participant received a laptop, headphones, and a closed envelope containing the paper-based post-test. The envelope was labelled with the participant identification number and a letter representing the experimental condition. Envelopes were randomly distributed to individual students and the researchers launched the appropriate condition of the computerized program for each student by typing in the participant id number and condition letter. The random envelope distribution ensured that the individual students were randomly distributed to the four experimental conditions. The researchers instructed students to begin the computerized program and students completed all portions of the program at their own pace. Once students had completed the program, the researchers instructed them to open the post-test envelope and answer all questions. The researchers then collected all laptops and post-test envelopes for scoring and data analysis.

\section{Results}


Statistical significance for all tests was set to an alpha level of .05, and when multiple comparisons were made, the Bonferroni adjustment was used. A preliminary Analysis of Variance (ANOVA) was conducted using pre-test scores as dependent variable and experimental condition as independent variable. The results from this analysis confirmed that there were no existing differences in prior knowledge among the experimental conditions, $F$ $<$ 1. A similar ANOVA revealed that that the instructional time spent on the introduction and practice session did not differ among experimental conditions, $F<1$. The following sections describe the results for each of the dependent variables in our study.

\subsection{Learning Outcomes}

To determine the impact of feedback schedule on the acquisition of problem-solving skills, we conducted an Analysis of Covariance (ANCOVA), using experimental condition as the independent variable, total post-test score as the dependent variable, and total pre-test score as covariate. Adjusted means and standard errors for all dependent variables, by experimental condition, are displayed in Table 2. The results indicated a significant effect of experimental condition on learners' post-test scores, $F(3,151)=6.82, M S E=4.76, p<.001$, $\eta_{p}^{2}=.12$. Follow-up comparisons among the experimental conditions revealed that the SFS condition had significantly higher post-test scores, compared to the SFP condition $(p=.001$; Cohen's $d=0.76)^{1}$. In addition, the TFS-P condition had significantly higher post-test scores, compared to both the SFP condition $(p<.001 ; d=0.97)$ and the TFP-S condition $(p=.008 ; d$ $=0.64)$. None of the remaining comparisons were statistically significant.

We also performed an ANCOVA, using total post-test score as the dependent variable, static or transitional feedback as independent variable, and pre-test score as covariate. The results indicated no significant difference between transitional feedback (i.e.,

\footnotetext{
${ }^{1}$ Note that significance levels for all follow-up comparisons were adjusted for multiple comparisons using the Bonferroni adjustment.
} 
the aggregate of the two transitional feedback conditions) and static feedback (i.e., the aggregate of the two static feedback conditions), $F<1$.

\subsection{Practice Problems}

We conducted two separate ANCOVAs, using the total practice score on the first two embedded practice problems (before feedback transitioned) and on the last two embedded practice problems (after feedback transitioned) as dependent variables, experimental condition as the independent variable, and pre-test scores as covariate. The analysis on the first two practice problems revealed a significant treatment effect, $F(3,151)=11.357, M S E=$ 2.20, $p<.001, \eta^{2} p=.18$. Follow-up pairwise comparisons revealed that the TFS-P condition had significantly higher practice scores on the first two problems, compared to both the SFP condition $(p<.001 ; d=0.97)$ and the TFP-S condition $(p<.001 ; d=0.87)$. Additionally, the SFS condition had higher scores on the first two practice problems, compared to the SFP condition $(p<.001 ; d=1.04)$ and the TFP-S condition $(p<.001 ; d=0.90)$.

The analysis on the last two practice problems revealed a significant treatment effect, $F(3,151)=4.52, M S E=1.91, p=.005, \eta^{2} p=.08$. Follow-up pairwise comparisons indicated that the TFS-P condition had significantly higher practice scores on the last two problems, compared to the SFP condition $(p=.004 ; d=0.64)$. Additionally, the SFS condition had higher scores, compared to the SFP condition $(p=.001 ; d=0.81)$. None of the remaining comparisons were statistically significant.

ANCOVAs were also performed for the first and last two practice problems, using transitional or static feedback as independent variable and pre-test score as covariate. Results indicated no significant difference between transitional feedback and static feedback for the first two problems $(F<1)$ or the last two problems $(F<1)$.

\subsection{Program Liking}


We conducted an ANCOVA, using experimental condition as independent variable average program liking rating as dependent variable, and pre-test score as covariate, to determine the effect of feedback transitioning on learners' perceptions of the program overall. Results indicated a significant treatment effect on learners' program ratings, $F(3,151)=3.71$, $M S E=0.86, p=.013, \eta_{p}^{2}=.07$. Follow-up comparisons among the experimental conditions revealed that learners in the TFS-P condition had significantly higher ratings of program liking, compared to the TFP-S condition $(p=.003 ; d=0.68)$. No other comparisons were statistically significant.

An ANCOVA was also conducted on feedback helpfulness ratings, using transitional or static feedback as independent variable and pre-test score as covariate. Results indicated no significant difference between transitional and static feedback $(F<1)$.

\subsection{Feedback Helpfulness}

We conducted an ANCOVA, using average ratings of feedback helpfulness as dependent variable, experimental condition as independent variable, and pre-test score as covariate to determine the effect of feedback transitioning on learners' perceptions of the helpfulness of feedback messages. The analysis indicated a significant effect of experimental condition on learners' ratings of feedback helpfulness, $F(3,151)=3.26, M S E=1.01, p=$ $.023, \eta^{2}{ }_{p}=.06$. Similar to the program liking measure, follow-up comparisons revealed a significant difference only between the TFS-P and TFP-S conditions. Learners in the TFS-P condition had significantly higher ratings of feedback helpfulness $(p=.004 ; d=0.63)$.

We also conducted an ANCOVA, using transitional and static feedback as independent variable, feedback helpfulness ratings as dependent variable, and pre-test scores as covariate. No significant difference was revealed between transitional and static feedback $(F<1)$.

\subsection{Cognitive Load}


An ANCOVA was used to determine the impact of feedback schedule on learners' perceptions of cognitive load, using pre-test scores as covariate. The analysis indicated a significant treatment effect on ratings of cognitive load, $F(3,151)=8.57, M S E=0.73, p<$ $.001, \eta_{p}^{2}=.15$. Follow-up pairwise comparisons revealed that cognitive load ratings were higher for learners in the SFP condition, compared to both the SFS condition $(p<.001 ; d=$ $1.14)$ and the TFS-P condition $(p=.003 ; d=0.70)$. Additionally, learners in the TFP-S condition had significantly higher cognitive load ratings than the SFS condition $(p<.001 ; d=$ 0.86). None of the remaining comparisons revealed significant differences. The cognitive load results support the feedback fading hypothesis (Section 1.4.1) in that the TFS-P condition had significantly lower cognitive load than the SFP condition; however the cognitive load ratings for the TFS-P condition were not significantly lower than for the TFPS or SFS conditions.

An ANCOVA was also conducted on feedback cognitive load ratings, using transitioning or static feedback as independent variable and pre-test score as covariate. No significant difference was revealed between transitioning and static conditions $(F<1)$.

\section{Discussion}

This study was conducted to examine the effects of transitional feedback schedules on learning and learners' perceptions. We compared post-test problem solving performance, practice problem performance, program ratings, and cognitive load perceptions of novice learners who learned electric circuit analysis using a computer-based learning environment with one of four types of feedback schedules: static feedback stepwise (SFS), static feedback problem (SFP), transitional feedback stepwise - problem (TFS-P), or transitional feedback problem - stepwise (TFP-S).

Comparisons of transitional feedback (i.e., aggregated measures from both transitional feedback conditions) with static feedback (aggregated measures from both static feedback 
conditions) did not reveal any significant differences. These comparison results indicate that transitional feedback does not have a general advantage over static feedback or vice versa. Rather, the specific schedule of the transitional or static feedback determines its effectiveness, as indicated by the one-way between subjects comparisons of the four specific examined feedback schedules.

Results revealed that students who either learned with stepwise feedback throughout (SFS), or transitioned from stepwise to problem feedback (TFS-P) had better near-transfer problem-solving performance, practice problem performance, and lower perceived cognitive load than students who learned with problem feedback throughout (SFP). Effect sizes for these comparisons (Cohen's $d$ ) indicated a strong effect when comparing the TFS-P condition to the SFP condition $(d=0.97)$ and a medium-strong effect when comparing the SFS condition to the SFP condition $(d=0.76)$. Students in the TFS-P condition also had better post-test performance than students in the TFP-S condition (a medium effect, $d=0.64$ ). Similarly, the TFS-P condition led to better general program liking ratings and ratings of feedback helpfulness, compared to the TFP-S condition. Moreover, cognitive load ratings were higher for the TFP-S condition compared to the SFS condition. Taken together, these results reveal a pattern of results that indicates support for the feedback fading hypothesis of Section 1.4.1. In particular, the results indicate that the most effective feedback schedule for learning the considered engineering problem-solving transitions students from stepwise to entire problem (summative) feedback, or employs stepwise feedback throughout. The inverse feedback fading hypothesis of Section 1.4.2 was not supported by the results.

A possible interpretation of these results is that novice learners, who have little knowledge about the considered engineering problem solving domain benefit from immediate feedback after each solution step. Immediate feedback after a solution step facilitates mental connections between the answer attempt and the feedback because the answer attempt is still 
in working memory when processing the feedback information. Additionally, low prior knowledge students can avoid cognitive overload by focusing attention on an individual solution step and the related feedback, rather than the whole problem and the corresponding feedback for all problem steps. The scores for the in-program practice problems further indicate the benefits of immediate feedback for novice learners. The practice scores for the first two problems were significantly higher for the SFS and TFS-P conditions, which provide immediate feedback after each solution step, compared to the SFP and TFP-S conditions, which provide feedback only after a complete problem.

Once students have developed relevant schemas to effectively solve similar problems, immediate feedback after every problem-solving step may not be necessary. The results for the scores of the last two practice problems, for which the TFS-P condition provided only feedback after a complete problem, indicate support for this interpretation. The learners in the TFS-P condition achieved equivalent practice scores on the last two problems as the learners in the SFS condition that received feedback after each problem step; and both TFS-P and SFS groups achieved significantly higher practice scores on the last two problems than the SFP group.

Interestingly, the cognitive load ratings for the TFS-P condition were not significantly lower than for the TFP-S condition, although the TFS-P condition resulted in significantly higher post-test scores. The lack of a significant cognitive load difference between the TFS-P and TFP-S conditions may be due to the employed cognitive load scale (Paas \& van Merrienboer, 1994), which commonly measures total cognitive load (de Jong, 2010; Schnotz \& Kürschner, 2007; Van Gog \& Paas, 2008). The TFS-P condition may have increased germane cognitive load while reducing extraneous cognitive load by giving feedback only after a completing a full problem for practice problems P3 and P4. Thus, similar to the recent study by Cierniak, Scheiter, and Gerjerts (2009), the total cognitive load may have remained 
at a relatively high level for the TFS-P condition. Consequently, no significant difference in the total cognitive load emerged between the TFS-P and TFP-S conditions, while the SPS condition resulted in significantly lower total cognitive load than the TFP-S condition. The development of reliable measures for distinguishing the different cognitive load types and employing such measures is an important direction for future research on feedback schedules.

Notably, the static stepwise (SFS) condition did not differ significantly from the TFSP condition on any of the dependant variables. Post-test performance was higher in the SFS condition, compared to the SFP condition. That is, when feedback schedules are not transitioned during a learning session, maintaining stepwise feedback throughout is more effective than summative (whole problem) feedback. However, with additional developing knowledge of the domain, feedback delivered immediately after every problem solving step could become disruptive to learning because students are required to mentally process new (and extraneous) information rather than continuing to the next step. Learners in more advanced stages of learning may have already developed problem-solving schemas; thus, presenting immediate feedback after each problem solving step could unnecessarily disrupt their problem-solving process, creating extraneous processing demands and hindering learning (Fyfe, Rittle-Johnson, \& DeCaro, 2012; Kalyuga, 2007; Kelley \& McLaughlin, 2012; Kester \& Kirschner, 2009). This study found no significant difference between the TFS-P and SFS conditions, indicating that in the examined engineering problem solving instruction, the feedback after each problem step did not significantly disrupt learning for the novice students.

Overall, the results of this study suggest that stepwise feedback in early stages of learning, followed by summative feedback in later stages is as effective as stepwise feedback throughout a learning session for the examined multi-step engineering problem solving. Thus, 
this study indicates that frequent, stepwise feedback is critical for the initial learning of multistep engineering problem solving by novice students.

\section{Limitations and Future Directions}

A limitation of this study is that transitional feedback was examined in the context of practice problems that required the learners to attempt all steps. Fading strategies that gradually reduce the number of worked problem steps and increase the number of problem steps attempted by the learner have been demonstrated to benefit novice learners in skill acquisition (Atkinson, Renkl, \& Merrill, 2003; Reisslein, Seeling, \& Reisslein, 2006; Renkl, Atkinson, Maier, \& Staley, 2002). Existing fading studies have considered static feedback for each problem solving step. An interesting future research direction is to combine the fading of worked problem steps with transitional feedback schedules that manipulate the feedback schedules for the problem steps that learners attempt within a fading of worked example steps design.

A related limitation of the present study is that akin to the studies on sequencing worked examples and practice problems (Leppink, et al., 2014; Van Gog, et al., 2011), we examined "abrupt" transitions, i.e., learners either received feedback on each problem step or only on the entire practice problem. Analogous to fading of worked example steps (Atkinson, et al., 2003; Renkl, et al., 2002) that smoothly transitions learners from worked examples to practice problems, future research could examine smooth feedback transition schedules that gradually reduce the frequency of feedback in the TFS-P design.

A further limitation of this study is that only rigid (non-adaptive) feedback transition schedules were examined. Transitioning feedback may be further promoted when students' understanding is assessed using embedded practice problems (Billings, 2012; Landsberg, et al., 2012). Stepwise feedback could be employed until a certain threshold of practice performance is obtained, at which point the learning environment would shift to summative 
feedback. Such adaption of the feedback schedule to the individual learner may have further beneficial effects for the stepwise - summative/problem feedback transitioning.

The study is limited in its use of a single educational domain (electric circuits), with a particular student population (high school students), using an immediate near-transfer test. Follow-up studies should examine feedback schedules using a different subject matter, use far-transfer test items, or administer delayed post-test to assess enduring impacts on problemsolving.

Due to the substantial time and resource demands for instructional experiments with pre-college students in their classrooms, we adopted an economical four condition experimental design. The classroom experiments provide for high external validity of the study, however, with a limited subject population of 156 students and four considered conditions, the present study had limited statistical power. Nevertheless, several significant large effects were uncovered, contributing a substantial set of original research results to the nascent research area of transitional feedback schedules. Future research may conduct followup comparisons of specific pairings of feedback conditions, such as static feedback stepwise (SFS) vs. transitional stepwise to problem feedback (TFS-P) with similar subject populations to examine such specific pairwise comparisons with larger statistical power. Also, the present study was limited to examining novice learners. An interesting future research direction is to examine novice learners with low prior knowledge as well as advanced learners with high prior knowledge. High prior knowledge learners may experience significant disruptive effects if stepwise feedback is maintained throughout the learning session (SFS) and may thus benefit significantly from feedback fading (TFS-P).

\section{References}

Anderson, J. R.. (1993). Rules of the mind. Hillsdale, NJ: Lawrence Erlbaum. 
Atkinson, R.K., Derry, S.J., Renkl, A., Wortham, D. (2000). Learning from examples: Instructional principles from the worked examples research. Review of Educational Research, 70, 181-214.

Atkinson, R. K., Renkl, A., \& Merrill, M.M. (2003). Transitioning from studying examples to solving problems: Effects of self-explanation prompts and fading worked-out steps. Journal of Educational Psychology, 95, 774-783.

Azevedo, R., and R.M. Bernard. (1995). A meta-analysis of the effects of feedback in computer-based instruction. Journ. Educational Computing Research,13(2): 111-27.

Baddeley, A. (1992). Working memory. Science, 255(5044), 556-559.

Bangert-Drowns, R.L., Kulik, C.C. Kulik, J.A. \& Morgan, M.T. (1991). The instructional effect of feedback in test-like events. Review of Educational Research, 61(2): 213-38.

Billings, D. R. (2012). Efficacy of adaptive feedback strategies in simulation-based training. Military Psychology, 24(2), 114-133.

Biesinger, K., \& Crippen, K. (2010). The effects of feedback protocol on self-regulated learning in a web-based worked example learning environment. Computers \& Education, 55, 1470-1482.

Butki, B. D., \& Hoffman, S. J. (2003). Effects of reducing frequency of intrinsic knowledge of results on the learning of a motor skill. Percept. and Motor Skills, 97(2), 569-580.

Butler, R. (1987). Task-involving and ego-involving properties of evaluation: Effects of different feedback conditions on motivational perceptions, interest, and performance. Journal of Educational Psychology, 79, 474-482.

Butler, A. C., \& Roediger, H. L. (2008). Feedback enhances the positive effects and reduces the negative effects of multiple-choice testing. Memory \& Cognition, 36(3), 604-616. 
Cierniak, G., Scheiter, K., \& Gerjets, P. (2009). Explaining the split-attention effect: Is the reduction of extraneous cognitive load accompanied by an increase in germane cognitive load? Computers in Human Behavior, 25, 315-324.

Clark, K., and Dwyer, F. (1998). Effect of different types of computer-assisted feedback strategies on achievement and response confidence. International Journal of Instructional Media, 25(1): 55-63.

Corbalan, G., Paas, F., \& Cuypers, H. (2010). Computer-based feedback in linear algebra: Effects on transfer performance and motivation. Comp. \& Educ., 55, 692-703.

De Jong, T. (2010). Cognitive load theory, educational research, and instructional design: some food for thought. Instructional Science, 38(2), 105-134.

Dihoff, R. E., Brosvic, G. M., Epstein, M. L., \& Cook, M. J. (2004). Provision of feedback during preparation for academic testing: Learning is enhanced by immediate but not delayed feedback. Psychological Record, 54(2), 207-232.

Earley, P.C., Northcraft, G.B., Le, C. \& Lituchy, T.R. (1990). Impact of process and outcome feedback on the relation of goal setting to task performance. Academy of Management Journal, 33(1), 87-105.

Fyfe, E. R., Rittle-Johnson, B., \& DeCaro, M. S. (2012). The effects of feedback during exploratory mathematics problem solving: Prior knowledge matters. Journal of Educational Psychology, 104(4), 1094.

Ginns, P. (2006). Integrating information: A meta-analysis of the spatial contiguity and temporal contiguity effects. Learning and Instruction, 16, 511-525.

Goodman, J. S., \& Wood, R. E. (2009). Faded versus increasing feedback, task variability trajectories, and transfer of training. Human Performance, 22, 64-85. 
Goodman. J.S., Wood, R.E., \& Chen, Z. (2011). Feedback specificity, information processing, and transfer of training. Organizational Behavior and Human Decision Processes, 115(2), 253-267.

Hattie, J. A. (1999). Influences on Student Learning. Inaugural professorial address, University of Auckland, New Zealand. Available at: http://wwwrp0a.teacherstoolbox.co.uk/downloads/managers/Influencesonstudent.pdf.

Hattie, J., \& Timperley, H. (2007). The power of feedback. Rev. Educ. Res., 77(1), 81-112.

Ho, L., \& Shea, J. B. (1978). Effects of relative frequency of knowledge of results on retention of a motor skill. Perceptual and Motor Skills, 46, 859-866.

Hsieh, I.G., and O’Neil Jr., H.F. (2002). Types of feedback in a computer-based collaborative problem-solving group task. Computers in Human Behavior, 18, 699-715.

Irwin, D. \& Nelms, M.R. (2008). Basic engineering circuit analysis, 8th Ed., Hoboken, NJ: John Wiley \& Sons Inc.

Johnson, D.S., Perlow, R. \& Pieper, K.F. (1993). Differences in task performance as a function of type of feedback: Learning-oriented versus performance oriented feedback. Journal of Applied Social Psychology, 23, 303-320.

Johnson, A.M., Reisslein, J., \& Reisslein, M. (2014). Representation sequencing in computerbased engineering education. Computers \& Education, 72, 249-261.

Kalyuga, S. (2007). Expertise reversal effect and its implications for learner-tailored instruction. Educational Psychology Review, 19(4), 509-539.

Kelley, C. M., \& McLaughlin, A. C. (2012). Individual differences in the benefits of feedback for learning. Human Factors, 54, 26-35.

Kester, L., \& Kirschner, P. A. (2009). Effects of fading support on hypertext navigation and performance in student-centered e-learning environments. Interactive Learning Environments, 17(2), 165-179. 
Kester, L., Kirschner, P. A., \& van Merriënboer, J. J. (2006). Just-in-time information presentation: Improving learning a troubleshooting skill. Contemporary Educational Psychology, 31(2), 167-185.

Kluger, A. N., \& DeNisi, A. (1996). The effects of feedback interventions on performance: a historical review, a meta-analysis, and a preliminary feedback intervention theory. Psychological Bulletin, 119(2), 254-284.

Kulhavy, R. W., \& Stock, W. A. (1989). Feedback in written instruction: The place of response certitude. Educational Psychology Review, 1(4), 279-308.

Kulik, J. A., \& Kulik, C. L. C. (1988). Timing of feedback and verbal learning. Review of Educational Research, 58(1), 79-97.

Laird, J. E., Rosenbloom, P. S., \& Newell, A. (1986). Chunking in Soar: The anatomy of a general learning mechanism. Machine Learning, 1(1), 11-46.

Lam, C. F., DeRue, D. S., Karam, E. P., \& Hollenbeck, J. R. (2011). The impact of feedback frequency on learning and task performance: Challenging the "more is better" assumption. Organiz. Behavior and Human Decision Processes, 116(2), 217-228.

Landsberg, C. R., Astwood Jr, R. S., Van Buskirk, W. L., Townsend, L. N., Steinhauser, N. B., \& Mercado, A. D. (2012). Review of Adaptive Training System Techniques. Military Psychology, 24(2), 96-113.

Leppink, J., Paas, F., van Gog, T., van der Vleuten, C. P., \& van Merriënboer, J. J. (2014). Effects of pairs of problems and examples on task performance and different types of cognitive load. Learning and Instruction, 30, 32-42.

Lewis, M. W., \& Anderson, J. R. (1985). Discrimination of operator schemata in problem solving: Learning from examples. Cognitive Psychology, 17(1), 26-65. 
Lin, L., Atkinson, R. K., Christopherson, R. M., Joseph, S. S., \& Harrison, C. J. (2013). Animated agents and learning: Does the type of verbal feedback they provide matter? Computers \& Education, 67, 239-249.

Lin, J.-W., Lai, Y.-C., \& Chuang, Y.-S. (2013). Timely Diagnostic Feedback for Database Concept Learning. Educational Technology \& Society. 16 (2), 228-242.

Lurie, N. H., \& Swaminathan, J. M. (2009). Is timely information always better? The effect of feedback frequency on decision making. Organizational Behavior and Human Decision Processes, 108(2), 315-329;

Maddox, W. T., Ashby, F. G., \& Bohil, C. J. (2003). Delayed feedback effects on rule-based and information-integration category learning. Journal of Experimental Psychology: Learning, Memory, and Cognition, 29(4), 650.

Maddox, W. T., \& Ing, A. D. (2005). Delayed feedback disrupts the procedural-learning system but not the hypothesis-testing system in perceptual category learning. Journal of Experimental Psychology: Learning, Memory, and Cognition, 31(1), 100-107.

Mason, J.B., and Bruning, R. (2001). Providing feedback in computer-based instruction: What the research tells us. Manuscript retrieved June 29, 2013 from: http://dwb.unl.edu/Edit/MB/MasonBruning.html.

Mason, L., Pluchino, P., Tornatora, M. C., \& Ariasi, N. (2013). An eye-tracking study of learning from science text with concrete and abstract illustrations. The Journal of Experimental Education, 81, 356-384.

Mathan, S. \& Koedinger, K.R. (2003). Recasting the feedback debate: Benefits of tutoring error detection and correction skills. Artificial Intelligence in Education, Hoppe, U., Verdejo, F. \& Kay, J. (Eds.), Amsterdam, The Netherlands: IOS Press.

Mayer, R. E., \& Moreno, R. (2003). Nine ways to reduce cognitive load in multimedia learning. Educational Psychologist, 38(1), 43-52. 
McDermott, L.C. \& Shaffer, P.S. (1992). Research as a guide for curriculum development: An example from introductory electricity. Part I: Investigation of student understanding, Am. J. Physics, 60, 994-1003.

McGuinness, C. (1990). Talking about thinking: The role of metacognition in teaching thinking. In K. Gilhooly, M. T. G. Keane, R. H. Logie, \& G. Erdos (Eds.). Lines of thinking (pp. 301-312). Chinchester: John Wiley \& Sons.

Moreno, R. (2004). Decreasing cognitive load for novice students: Effects of explanatory versus corrective feedback in discovery-based multimedia. Instructional Science, $32(1-2), 99-113$.

Moreno, R., \& Durán, R. (2004). Do Multiple Representations Need Explanations? The Role of Verbal Guidance and Individual Differences in Multimedia Mathematics Learning. Journal of Educational Psychology, 96(3), 492-503.

Moreno, R., \& Mayer, R. (2007). Interactive multimodal learning environments. Educational Psychology Review, 19(3), 309-326.

Moreno, R., Reisslein, M., \& Ozogul, G. (2009). Optimizing Worked-Example Instruction in Electrical Engineering: The Role of Fading and Feedback during Problem-Solving Practice. Journal of Engineering Education, 98(1), 83-92.

Mory, E. H. (2004). Feedback research revisited. Handbook of Research on Educational Communications and Technology, 2, 745-783.

Munro, A., Fehling, M. R., Towne, D. M. (1985). Instruction intrusiveness in dynamic simulation training. Journal of Computer-Based Instruction, 12(2), 50-53.

Narciss, S., Sosnovsky, S., Schnaubert, L., Andrès, E., Eichelmann, A., Goguadze, G., \& Melis, E. (2013). Exploring feedback and student characteristics relevant for personalizing feedback strategies, Computers \& Education, 71, 56-76. 
Ozogul, G., Johnson, A.M., Moreno, R., \& Reisslein, M. (2012). Technological literacy learning with cumulative and stepwise integration of equations into electrical circuit diagrams, IEEE Transactions on Education, 55, 480-487.

Paas, F. G. W. C., \& van Merrienboer, J. J. G. (1994). Variability of worked examples and transfer of geometrical problem-solving skills: A cognitive load approach. Journal of Educational Psychology, 86(1), 122-133.

Pintrich, P. R. (2003). A motivational science perspective on the role of student motivation in learning and teaching contexts. Journal of Educational Psychology, 95(4), 667-686.

Reisslein, J., Johnson, A.M., \& Reisslein, M. (2014). Color coding of circuit quantities in introductory circuit analysis instruction, IEEE Transactions on Education, in print, 2014.

Reisslein, M., Moreno, R., \& Ozogul, G. (2010). Pre-college electrical engineering instruction: The impact of abstract vs. contextualized representation and practice on learning. Journal of Engineering Education, 99(3), 225-235.

Reisslein, J., Seeling, P., \& Reisslein, M. (2006). Comparing static fading with adaptive fading to independent problem solving: The impact on the achievement and attitudes of high school students learning electrical circuit analysis, Journal of Engineering Education, 95, 217-226.

Renkl, A., \& Atkinson, R. K. (2003). Structuring the transition from example study to problem solving in cognitive skill acquisition: A cognitive load perspective. Educational Psychologist, 38(1), 15-22.

Renkl, A., Atkinson, R. K., Maier, U. H., \& Staley, R. (2002). From example study to problem solving: Smooth transitions help learning. J. Exp. Educ., 70(4), 293-315.

Schnotz, W., \& Kürschner, C. (2007). A reconsideration of cognitive load theory. Educational Psychology Review, 19(4), 469-508. 
Schooler, L. J., \& Anderson, J. R. (2008). The disruptive potential of immediate feedback. Department of Psychology. Paper 27. Available at: http://repository.cmu.edu/psychology/27

Schoppek, W., \& Tulis, M. (2010). Enhancing arithmetic and word-problem solving skills efficiently by individualized computer-assisted practice. J. Educ. Res., 103, 239-252.

Shute, V. J. (2008). Focus on formative feedback. Rev. Educ. Research, 78(1), 153-189.

Skromme, B.J., Wang, Q., Reyes, P., Quick, J.M., Atkinson, R.K., \& Frank, T. (2013) Teaching linear circuit analysis techniques with computers, Proc. ASEE.

Smith, T. A., \& Kimball, D. R. (2010). Learning from feedback: Spacing and the delayretention effect. J. Exp. Psych.: Learning, Memory, and Cognition, 36(1), 80-95.

Swinnen, S. P., Schmidt, R. A., Nicholson, D. E., \& Shapiro, D. C. (1990). Information feedback for skill acquisition: Instantaneous knowledge of results degrades learning. J. Experimental Psychology: Learning, Memory, and Cognition, 16(4), 706-716.

Sweller, J. (1999). Instructional design in technical areas. Melbourne, Austr.: ACER Press.

Sweller, J., van Merriënboer, J., \& Paas, F. (1998). Cognitive architecture and instructional design. Educational Psychology Review, 10, 251-296.

Tulving, E. (1977). Episodic and semantic memory. In E. Tulving and W. Donaldson (Eds.), Organization of memory (pp. 381-403). New York: Academic Press.

Van der Kleij, F. M., Eggen, T. J., Timmers, C. F., \& Veldkamp, B. P. (2012). Effects of feedback in a computer-based assessment for learning. Comp. \& Educ., 58, 263-272.

Van Duyne, L. R., Jentsch, K., Bowers, C., Burroughs, W., Cannon-Bowers, J., \& Salas, E. (2001, October). Comparing the utility and sequencing of different types of feedback. In Proceedings of the Human Factors and Ergonomics Society Annual Meeting (Vol. 45, No. 26, pp. 1843-1847). SAGE Publications. 
Van Gog, T., Kester, L., \& Paas, F. (2011). Effects of worked examples, example-problem, and problem-example pairs on novices' learning. Contemporary Educational Psychology, 36(3), 212-218.

Van Gog, T., \& Paas, F. (2008). Instructional efficiency: Revisiting the original construct in educational research. Educational Psychologist, 43, 16-26.

Winstein, C. J., \& Schmidt, R. A. (1990). Reduced frequency of knowledge of results enhances motor skill learning. Journal of Experimental Psychology: Learning, Memory, and Cognition, 16(4), 677-691.

Wulf, G., \& Schmidt, R. A., (1989). The learning of generalized motor programs: Reducing the relative frequency of knowledge of results enhances memory. Journal of Experimental Psychology: Learning, Memory, and Cognition, 15(4), 748-757.

Wulf, G., \& Shea, C. H. (2002). Principles derived from the study of simple skills do not generalize to complex skill learning. Psychonomic Bulletin \& Review, 9(2), 185-211.

Yang, D., Streveler, R. A., Miller, R. L., Slotta, J. D., Matusovich, H. M., \& Magana, A. J. (2012). Using computer-based online learning modules to promote conceptual change: Helping students understand difficult concepts in thermal and transport science. International Journal of Engineering Education, 28(3), 686-700. 
Figure 1. Screen shot from the practice session of the computer-based learning environment at the instant when the learner begins to attempt solving the first practice problem step.

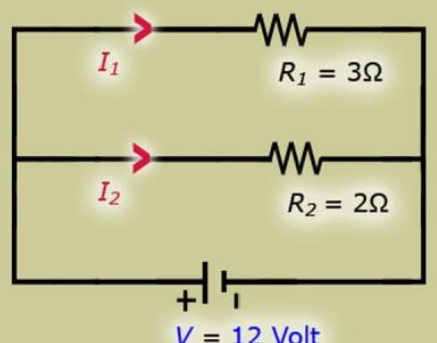

Problem Text

You have a parallel circuit with two resistors; $R_{1}=3 \mathrm{Ohm}$ and $R_{2}=2 \mathrm{Ohm}$. The resistors are connected to a voltage source with $V=12$ Volts. What is the total resistance, $R_{\text {total }}$ of this parallel circuit?

$\begin{array}{lll}\text { First } & \text { Calculate individual currents flowing through the resistors. }\end{array}$

Solution Step 
Figure 2. Screen shot from condition with stepwise feedback at the instant after the learner had completed the solution attempt of the first practice problem step, studied the feedback, and proceeded to attempting the second solution step. The learning environment provided corrective feedback in the form of a watermark (red cross for incorrect attempt, green checkmark for correct attempt) as well as explanatory feedback.

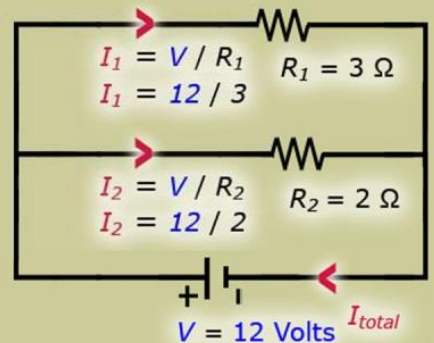

Problem Text

You have a parallel circuit with two resistors; $R_{1}=3 \mathrm{Ohm}$ and $R_{2}=2 \mathrm{Ohm}$. The resistors are connected to a voltage source with $V=12$ Volts. What is the total resistance, $R_{\text {total }}$ of this parallel circuit?

\begin{tabular}{|c|c|c|c|}
\hline \multirow{2}{*}{$\begin{array}{c}\text { First } \\
\text { Solution Step }\end{array}$} & \multicolumn{3}{|c|}{ Calculate individual currents flowing through the resistors. } \\
\hline & $\begin{array}{ll}I_{1}=1 \quad \text { Ampere } \\
I_{2}=2 \quad \text { Ampere }\end{array}$ & $\begin{array}{l}\text { Current flowing through } R_{1} \text { is } I_{1}=V / R_{1} \\
\text { Current flowing through } R_{2} \text { is } I_{2}=V / R_{2}\end{array}$ & $\begin{array}{l}I_{1}=12 / 3=4 \text { Ampere } \\
I_{2}=12 / 2=6 \text { Ampere }\end{array}$ \\
\hline $\begin{array}{l}\text { Second } \\
\text { Solution Step }\end{array}$ & \multicolumn{3}{|l|}{$\begin{array}{l}\text { Calculate the total current flow. } \\
\text { ( Please enter the numerical answer below ) }\end{array}$} \\
\hline & Ampere & & \\
\hline
\end{tabular}


Figure 3. Screen shot from condition with problem (summative) feedback at the instant after the learner had completed the solution attempt of the first practice problem step and proceeded to attempting the second solution step. The learning environment prompts the learner to attempt the second practice problem step without providing feedback on the solution attempt of the first practice problem step. Feedback for the entire practice problem, i.e., all practice problem steps, is provided after the learner has attempted all practice problem steps.

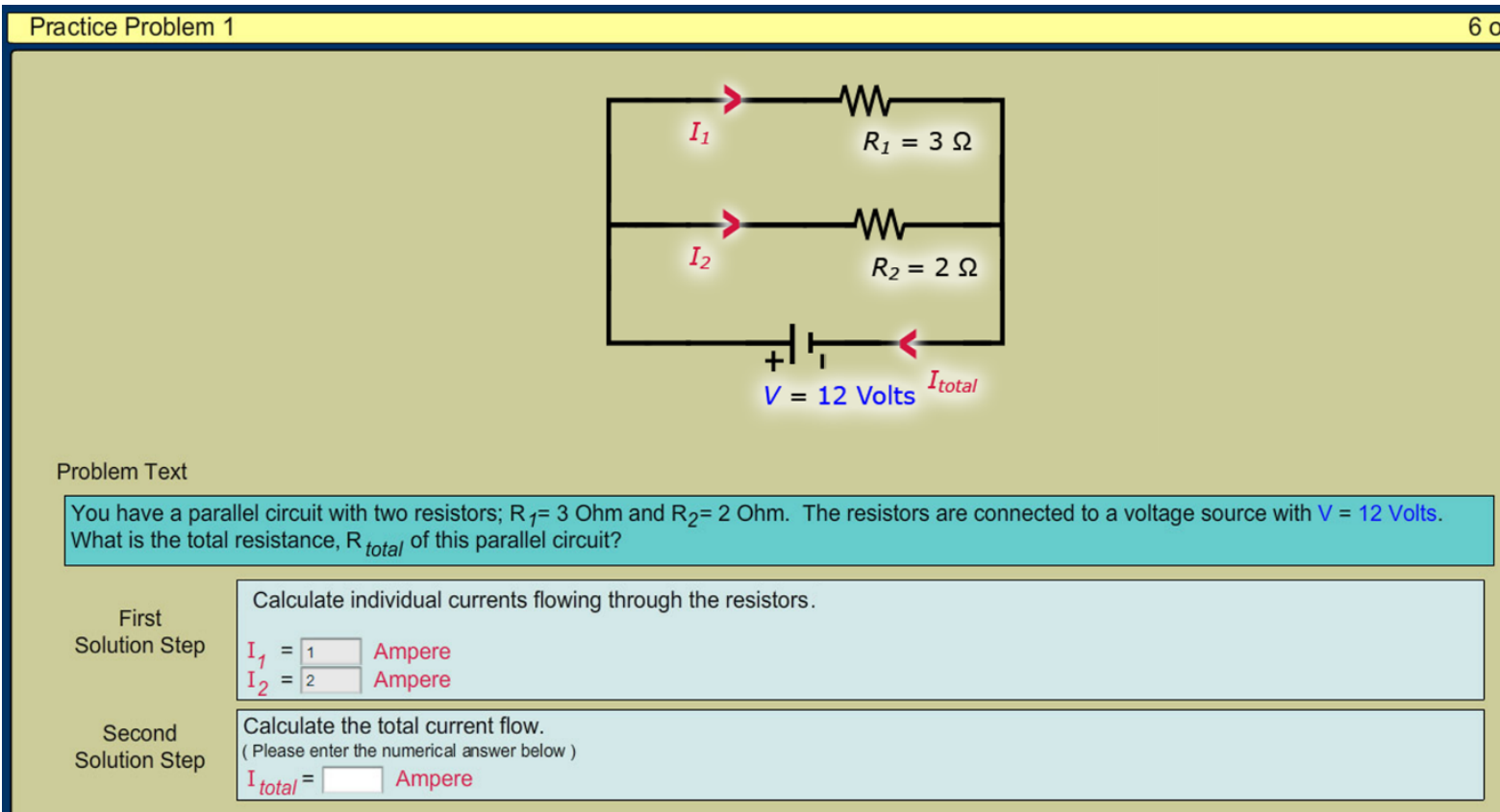


Table 1

Practice Problem Feedback Schedules, by Experimental Condition

\begin{tabular}{|c|c|c|c|c|}
\hline \multirow[t]{2}{*}{ Feedback } & \multicolumn{4}{|c|}{ Practice Problem } \\
\hline & $\mathrm{P} 1$ & $\mathrm{P} 2$ & $\mathrm{P} 3$ & $\mathrm{P} 4$ \\
\hline $\begin{array}{l}\text { Static, stepwise } \\
\text { (SFS) }\end{array}$ & $\begin{array}{l}\text { S1-FS } \\
\text { S2-FS } \\
\text { S3-FS }\end{array}$ & $\begin{array}{l}\text { S1-FS } \\
\text { S2-FS } \\
\text { S3-FS }\end{array}$ & $\begin{array}{l}\text { S1-FS } \\
\text { S2-FS } \\
\text { S3-FS }\end{array}$ & $\begin{array}{l}\text { S1-FS } \\
\text { S2-FS } \\
\text { S3-FS }\end{array}$ \\
\hline $\begin{array}{l}\text { Static, problem } \\
\text { (SFP) }\end{array}$ & $\begin{array}{l}\text { S1 } \\
\text { S2 } \\
\text { S3 } \\
\text { FP }\end{array}$ & $\begin{array}{l}\text { S1 } \\
\text { S2 } \\
\text { S3 } \\
\text { FP }\end{array}$ & $\begin{array}{l}\text { S1 } \\
\text { S2 } \\
\text { S3 } \\
\text { FP }\end{array}$ & $\begin{array}{l}\text { S1 } \\
\text { S2 } \\
\text { S3 } \\
\text { FP }\end{array}$ \\
\hline $\begin{array}{l}\text { Transitional, } \\
\text { step - problem } \\
\text { (TFS-P) }\end{array}$ & $\begin{array}{l}\text { S1-FS } \\
\text { S2-FS } \\
\text { S3-FS }\end{array}$ & $\begin{array}{l}\text { S1-FS } \\
\text { S2-FS } \\
\text { S3-FS }\end{array}$ & $\begin{array}{l}\text { S1 } \\
\text { S2 } \\
\text { S3 } \\
\text { FP }\end{array}$ & $\begin{array}{l}\text { S1 } \\
\text { S2 } \\
\text { S3 } \\
\text { FP }\end{array}$ \\
\hline $\begin{array}{l}\text { Transitional, } \\
\text { problem - step } \\
(\text { TFP-S) }\end{array}$ & $\begin{array}{l}\text { S1 } \\
\text { S2 } \\
\text { S3 } \\
\text { FP }\end{array}$ & $\begin{array}{l}\text { S1 } \\
\text { S2 } \\
\text { S3 } \\
\text { FP }\end{array}$ & $\begin{array}{l}\text { S1-FS } \\
\text { S2-FS } \\
\text { S3-FS }\end{array}$ & $\begin{array}{l}\text { S1-FS } \\
\text { S2-FS } \\
\text { S3-FS }\end{array}$ \\
\hline
\end{tabular}

The problem-solving practice consisted of four practice problems. Steps S1, S2, and S3 required a solution attempt by the learner. "FS" denotes feedback for a given step and "FP" denotes feedback for an entire problem. 
Table 2

Adjusted Means and Standard Errors for Pre-test Scores, Post-test Scores, Practice Scores on P1 and P2 (before feedback transition) and P3 and P4 (after feedback transition), Program Liking Ratings, Feedback Helpfulness Ratings, and Cognitive Load Ratings, by Experimental Condition

\begin{tabular}{lccccccc}
\hline & $\begin{array}{c}\text { Pre-test } \\
(\max =6)\end{array}$ & $\begin{array}{c}\text { Post-test } \\
(\max =12)\end{array}$ & $\begin{array}{c}\text { Practice 1,2 } \\
(\max =6)\end{array}$ & $\begin{array}{c}\text { Practice 3, 4 } \\
(\max =6)\end{array}$ & $\begin{array}{c}\text { Program } \\
\text { Liking }\end{array}$ & $\begin{array}{c}\text { Feedback } \\
\text { Helpfulness }\end{array}$ & $\begin{array}{c}\text { Cognitive } \\
\text { Load }\end{array}$ \\
SFS $(\mathrm{N}=38)$ & $2.63(0.24)$ & $9.49(0.35)^{1}$ & $3.71(0.24)^{1,2}$ & $4.68(0.22)^{1}$ & $3.65(0.15)$ & $3.63(0.16)$ & $2.12(0.14)$ \\
SFP $(\mathrm{N}=38)$ & $2.61(0.19)$ & $7.75(0.35)$ & $2.45(0.24)$ & $3.63(0.22)$ & $3.38(0.15)$ & $3.87(0.16)$ & $2.96(0.14)^{3,4}$ \\
TFS-P (N = 41) & $2.71(0.19)$ & $9.61(0.34)^{1,2}$ & $3.76(0.23)^{1,2}$ & $4.54(0.22)^{1}$ & $3.85(0.15)^{2}$ & $4.00(0.16)^{2}$ & $2.38(0.13)$ \\
TFP-S $(\mathrm{N}=39)$ & $2.72(0.18)$ & $8.30(0.35)$ & $2.27(0.24)$ & $4.08(0.22)$ & $3.21(0.15)$ & $3.34(0.16)$ & $2.89(0.14)^{3}$ \\
\hline
\end{tabular}

Notes $(p<.05)$ :

${ }^{1}$ Significantly higher than SFP condition

${ }^{2}$ Significantly higher than TFP-S condition

${ }^{3}$ Significantly higher than SFS condition

${ }^{4}$ Significantly higher than TFS-P condition

- An experiment investigated feedback schedules in problem solving instruction

- $\quad$ Static stepwise feedback outperformed problem (summative) feedback on posttest

- $\quad$ Stepwise-problem feedback schedule outperformed problem-stepwise feedback

- Initial stepwise feedback is critical for problem-solving learning by novices 